prevalence was $5.8 \%(3.9-7.7 \%)$ among all those with no qualifications and the SII had increased from 1.0, in 1995, to 3.9; (from 1.8 to 5.1 among women). The prevalence of obesity increased across the surveys with minimal change in the inequality gap. among those with no qualifications the prevalence increased from $23.3 \%$ (21.2-25.3\%) at baseline to $31.2 \%(26.5-35.8 \%)$ in 2008 . In the corresponding years the SII for obesity had increased from 10.7 to 13.0. Difficulties in reporting alcohol consumption trends arise from changes in recording practices between surveys; approaches will be presented.

Conclusions Individuals of lower socio-economic status continue to carry the heaviest burden of CVD risk factors. There has been little, if no reduction in the inequality gap over time; indeed for some factors it may be growing.

\section{RELATIVE IMPORTANCE OF SMOKING, PHYSICAL ACTIVITY AND SCREEN-BASED ENTERTAINIMENT IN EXPLAINING SOCIO-ECONOMIC INEQUALITIES IN CARDIOVASCULAR DISEASE RISK}

doi:10.1136/jech.2010.120956.32

M Roth, E Stamatakis. Health and Social Surveys Research Group, Department of Epidemiology and Public Health, UCL, London, UK

Objective To assess the extent to which smoking, moderate-tovigorous physical activity (MVPA), and screen-based entertainment (SBE) explain the association between socio-economic position (SEP) and CVD risk.

Design Cross-sectional health examination survey linked to mortality data.

Setting The Scottish Health Survey 2003 is a survey of a random sample of the general population living in private households in Scotland.

Participants The cross-sectional component of this study included 2782 adults aged 16 and over who had complete information on all socio-economic and clinical measures used to calculate the SEP score and the cardio-metabolic risk score. The longitudinal component of this study considered 4621 respondents, aged 35 and over who consented to having their records linked to National Health Service administrative data.

Main outcome measures We calculated the percentage of the association between lower SEP and CVD risk that smoking, MVPA, and SBE explain in two ways: a) cross-sectionally using a cardio-metabolic risk score (based on total cholesterol, HDL cholesterol, HbA1c, C-reactive protein, BMI, waist, hypertension) dichotomized as three or more / less than three risk factors as the main outcome, and b) longitudinally with CVD (fatal/non-fatal) events as the main outcome. The main exposure variable in both sets of analyses was a composite SEP score (based on social class, income, and education). A total of 179 incident cardiovascular events including deaths, which occurred over 19864 person years, an average of 4.3 years, was used in the analysis.

Results In both sets of analyses, SBE explained a larger percentage of the association between SEP and CVD risk than either smoking or MVPA. In the cross-sectional analysis, SBE accounted for $30.0 \%$ of the association between lowest SEP and having a cardio-metabolic risk score of three or more, followed by MVPA (16.4\%) and smoking (10.9\%). A similar pattern emerged from the longitudinal analysis, where SBE emerged as the largest contributor, accounting for $30.4 \%$, to explaining the association between lowest SEP and increased risk of having a CVD event. Smoking explained the next highest percentage (26.7\%) and MVPA the least (14.6\%). The fully adjusted model with all three variables explained $52.5 \%$ of the relationship. Conclusion Since SBE explains a larger proportion of the association between SEP and CVD risk than smoking or MVPA, public health policies aimed at reducing inequalities in health should include guidance on reductions of sedentary behaviour in addition to guidance already available on smoking cessation and the promotion of physical activity.

\section{SOCIO-ECONOMIC TRENDS IN CARDIOVASCULAR RISK FACTORS IN ENGLAND, 1994-2008}

doi:10.1136/jech.2010.120956.33

${ }^{1} \mathrm{~S}$ Scholes, ${ }^{1} \mathrm{M}$ Bajekal, ${ }^{1} \mathrm{R}$ Raine, ${ }^{2} \mathrm{M}$ O'Flaherty, ${ }^{2} \mathrm{~S}$ Capewell. ${ }^{1}$ Department of Epidemiology and Public Health, University College London, UK; ${ }^{2}$ Division of Public Health, University of Liverpool, UK

Objective Recent large falls in Coronary Heart Disease (CHD) mortality rates have been attributed to reductions in behavioural and physiological risk factors, particularly smoking, cholesterol and high systolic blood pressure (SBP), and also to the increasingly widespread use of cardiological treatments. Such gains, however, have been partially offset by unfavourable trends in Body Mass Index (BMI), diabetes and physical inactivity, possibly exacerbating inequalities. Using data from the Health Survey for England from 1994 to 2007, we therefore examined differentials in CHD risk factors across socio-economic groups over recent years.

Methods The Health Survey for England (HSfE) is an annual, nationally representative health interview and examination survey containing a core element - which includes risk factors such as smoking and BMI as well as biomarkers like blood pressure and saliva cotinine - and a regularly repeated disease module. In 1998, 2003 and 2006 the HSfE focused on CHD risk factors. Socioeconomic circumstance (SEC) was defined by grouped quintiles of residential deprivation. A series of regression models were used to analyse the influence of SEC and time on risk factor levels, separately for each gender. Interaction terms were used to test whether risk factor trends differed between SEC groups.

Results SEC gradients in risk factors were most pronounced for current smoking, fruit and vegetable consumption, BMI (women only) and diabetes (women aged 55-74). Recent trends present a mixed picture. Smoking and SBP declined year-on-year for most SEC groups; cholesterol levels fell significantly between 2003 and 2006; and (beneficial) physical activity and fruit and vegetable consumption increased. However, mean BMI and diabetes prevalence among older age-groups increased across all SEC groups. Despite favourable trends in major risk factors across all social groups, the inequality gap remained essentially unchanged between 1994 and 2007

Conclusions Persistent SEC differentials in major risk factors (smoking and poor diet) highlight an important priority for more effective policies for healthy food and tobacco control. Furthermore, research is now crucial to quantify the extent to which these persistent inequalities in CHD risk factor levels might explain the substantial inequalities observed in CHD mortality.

\section{ASSOCIATION OF NEIGHBOURHOOD SOCIO-ECONOMIC STATUS AND INDIVIDUAL SOCIO-ECONOMIC STATUS WITH CARDIOVASCULAR RISK FACTORS IN AN EASTERN GERMAN POPULATION - THE CARLA STUDY 2002-2006}

doi:10.1136/jech.2010.120956.34

${ }^{1} \mathrm{~K}$ H Greiser, ${ }^{2} \mathrm{D}$ Tiller, ${ }^{2} \mathrm{O}$ Kuss, ${ }^{2} \mathrm{~A}$ Kluttig, ${ }^{3} \mathrm{~B}$ Schumann, ${ }^{4} \mathrm{~K}$ Werdan, ${ }^{2} \mathrm{~J}$ Haerting. ${ }^{1}$ German Cancer Research Center, Division of Cancer Epidemiology, Heidelberg, Germany; ${ }^{2}$ Institute of Medical Epidemiology, Biostatistics, and Informatics, MartinLuther-University Halle-Wittenberg, Halle (Saale), Germany; ${ }^{3}$ Federal Institute for Occupational Safety and Health (BAuA), Berlin, Germany; ${ }^{4}$ University Clinic for Internal Medicine III, Martin-Luther-University Halle-Wittenberg, Halle (Saale), Germany

Background/objectives Socio-economic status (SES) has long been recognized as determinant of cardiovascular risk factors and disease. Recent studies suggest an association of neighbourhood SES with 